\title{
Relating Some Nonlinear Systems to a Cold Plasma Magnetoacoustic System
}

\author{
Jennie D'Ambroise1, Floyd L. Williams² \\ ${ }^{1}$ Department of Mathematics/CIS, SUNY Old Westbury, Old Westbury, NY, USA \\ ${ }^{2}$ Department of Mathematics and Statistics, University of Massachusetts, Amherst, MA, USA \\ Email: dambroisej@oldwestbury.edu,williams@math.umass.edu
}

How to cite this paper: D'Ambroise, J. and Williams, F.L. (2020) Relating Some Nonlinear Systems to a Cold Plasma Magnetoacoustic System. Journal of Modern Physics, 11, 886-906.

https://doi.org/10.4236/jmp.2020.116054

Received: March 20, 2020

Accepted: June 2, 2020

Published: June 5, 2020

Copyright () 2020 by author(s) and Scientific Research Publishing Inc. This work is licensed under the Creative Commons Attribution International License (CC BY 4.0).

http://creativecommons.org/licenses/by/4.0/

\section{(c) (i) Open Access}

\begin{abstract}
Using a Gurevich-Krylov solution that describes the propagation of nonlinear magnetoacoustic waves in a cold plasma, we construct solutions of various other nonlinear systems. These include, for example, Madelung fluid, reaction diffusion, Broer-Kaup, Boussinesq, and Hamilton-Jacobi-Bellman systems. We also construct dilaton field solutions for a Jackiw-Teitelboim black hole with a negative cosmological constant. The black hole metric corresponds to a cold plasma metric by way of a change of variables, and the plasma dilatons and cosmological constant also have an expression in terms of parameters occurring in the Gurevich-Krylov solution. A dispersion relation, moreover, links the magnetoacoustic system and a resonance nonlinear Schrödinger equation.
\end{abstract}

\section{Keywords}

Cold Plasma, Magnetoacoustic Waves, Resonance Nonlinear Schrödinger Equation, Reaction Diffusion System, Jackiw-Teitelboim Black Hole, Dilaton Field, Ricci Scalar Curvature, Jacobi Elliptic Function

\section{Introduction}

Over the past years, points of connection of plasma physics to various nonlinear equations of significant importance have been explored. An initial connection can be traced back to H. Washimi and T. Taniuti [1], for example, who showed that the one-dimensional asymptotic behavior (as $t \rightarrow \infty$ ) of small amplitude ion-acoustic waves was described by the Korteweg-deVries equation-following on a parallel track work of C. S. Gardner and G. K. Morikawa [2]. The paper of A. Jeffrey [3] provides for some systematic details on this particular connection, and it includes remarks, for example, on the work of Y. A. Berezin and V. I. 
Karpman [4].

In the 2007 paper [5] of J.-H. Lee, O. K. Pashaev, C. Rogers, and W. K. Schief the resonance nonlinear Schrödinger (RNLS) equation

$$
i \psi_{t}+\psi_{x x}+\gamma|\psi|^{2} \psi=\delta \frac{|\psi|_{x x}}{|\psi|} \psi
$$

appears in connection with a discussion of one-dimensional, long magnetoacoustic waves in a cold plasma subject to a transverse magnetic field $\overrightarrow{\boldsymbol{B}}$. Here $\gamma, \delta \in \mathbb{R}=$ the field of real numbers with $\delta>1$, and $|\psi|_{x x} /|\psi|$ is a de Broglie quantum potential. For $\gamma=-1 / 2$, a complex-valued wave function solution $\psi$ of the form

$$
\psi(x, t)=\sqrt{\rho(x, t)} \mathrm{e}^{-i S(x, t)}
$$

was obtained, where $\rho>0$ is the plasma density and $S$ is a real-valued velocity potential. That is, the velocity field $u$ of the plasma is given by $u=-2 S_{x}$. In the present paper we consider for an arbitrary $\gamma<0$ solutions of Equation (1) of the form

$$
\psi=\mathrm{e}^{R-i S}
$$

for real-valued functions $R(x, t), S(x, t)$. Such a function $\psi$ is a solution if and only if the pair $(R, S)$ is a solution of the Madelung fluid system

$$
\begin{aligned}
& R_{t}-S_{x x}-2 R_{x} S_{x}=0 \\
& S_{t}+(1-\delta)\left[R_{x x}+R_{x}^{2}\right]-S_{x}^{2}+\gamma \mathrm{e}^{2 R}=0
\end{aligned}
$$

independently of the assumption $\gamma<0$.

The system of main interest for us is the nonlinear magnetoacoustic system (MAS)

$$
\begin{aligned}
& \rho_{t}+(\rho u)_{x}=0 \\
& u_{t}+u u_{x}+\rho_{x}+\beta^{2}\left[\frac{\rho_{x x}}{\rho}-\frac{1}{2}\left(\frac{\rho_{x}}{\rho}\right)^{2}\right]_{x}=0
\end{aligned}
$$

which describes the propagation of the aforementioned magnetoacoustic waves in the cold plasma, under some simplifying assumptions that include the uni-axial propagation assumption

$$
\overrightarrow{\boldsymbol{B}}(x, t)=B(x, t) \vec{k}, \vec{u}(x, t)=u(x, t) \vec{i} .
$$

Here $\beta>0$ and $\vec{i}, \vec{k}$ are unit vectors along the $x$ and $z$ axes. The system (5) is derived by way of a shallow water type approximation of the system

$$
\begin{aligned}
& \rho_{t}+(\rho u)_{x}=0 \\
& u_{t}+u u_{x}+\frac{B}{\rho} B_{x}=0 \\
& B=\rho+\left(\frac{1}{\rho} B_{x}\right)_{x}
\end{aligned}
$$

where a change of variables $(x, t) \rightarrow(\beta x, \beta t)$ is employed (under which the first 
two equations in (7) are invariant and third one becomes $\left.B=\rho+\beta^{2}\left(B_{x} / \rho\right)_{x}\right)$, and where an expansion of $B$ of the form

$$
B=\rho+\beta^{2}\left(\frac{\rho_{x}}{\rho}\right)_{x}+\mathcal{O}\left(\beta^{4}\right)
$$

is inserted into the second equation [5] [6] [7].

We will work with the following explicit traveling wave solution $(\rho>0, u)$ of the MAS (5) given by A. Gurevich and A. Krylov (G-K) in [8]. For a choice $u_{0}>0, \quad \alpha_{3}>\alpha_{2} \geq \alpha_{1} \geq 0$, define

$$
\begin{aligned}
& v \stackrel{\text { def }}{=} u_{0} / \beta>0, a \stackrel{\text { def }}{=}+\left(\alpha_{3}-\alpha_{1}\right)^{\frac{1}{2}} / 2 \beta>0, \quad \kappa \stackrel{\text { def }}{=}\left(\frac{\alpha_{3}-\alpha_{2}}{\alpha_{3}-\alpha_{1}}\right)^{\frac{1}{2}} \in(0,1), \\
& C \stackrel{\text { def }}{=}+\left(\alpha_{1} \alpha_{2} \alpha_{3}\right)^{\frac{1}{2}}=\left(\alpha_{1}\left[4 a^{2} \beta^{2}\left(1-\kappa^{2}\right)+\alpha_{1}\right]\left[4 a^{2} \beta^{2}+\alpha_{1}\right]\right)^{1 / 2}
\end{aligned}
$$

where the second expression for $C$ in (9) follows as $1-\kappa^{2} \stackrel{\text { def }}{=}\left(\alpha_{2}-\alpha_{1}\right) /\left(\alpha_{3}-\alpha_{1}\right)$ and $4 a^{2} \beta^{2} \stackrel{\text { def }}{=} \alpha_{3}-\alpha_{1} \Rightarrow\left[4 a^{2} \beta^{2}\left(1-\kappa^{2}\right)+\alpha_{1}\right]\left[4 a^{2} \beta^{2}+\alpha_{1}\right]=\alpha_{2} \alpha_{3}$. Then for the standard Jacobi elliptic function $d n(x, \kappa)$ with elliptic modulus $\kappa$ [9]

$$
\begin{aligned}
& \rho(x, t)=\alpha_{1}+4 a^{2} \beta^{2} d n^{2}(a(x-\beta v t), \kappa), \\
& u(x, t)=u_{0}+C / \rho(x, t) .
\end{aligned}
$$

The choice for $\delta>1$ in (1) will be $\delta \stackrel{\text { def }}{=} \beta^{2}+1$. As shown in [10] (also compare [5] [7]), given a solution $(\rho>0, u)$ of the MAS (5) and the assumption $\gamma<0$, one can construct a corresponding solution $(R, S)$ of the Madelung fluid system (4) where

$$
R \stackrel{\text { def }}{=} \frac{1}{2} \log \left(\frac{\rho}{-2 \gamma}\right), S_{x} \stackrel{\text { def }}{=}-\frac{u}{2}
$$

and, moreover, a corresponding solution $(r, s)$ of the reaction diffusion system (RDS)

$$
\begin{aligned}
& r_{t}-r_{x x}+B r^{2} s=0 \\
& s_{t}+s_{x x}-B r s^{2}=0
\end{aligned}
$$

where

$$
\begin{aligned}
& r(x, t) \stackrel{\text { def }}{=} \mathrm{e}^{R(x, t / \beta)} \mathrm{e}^{\phi(x, t)}, \\
& s(x, t) \stackrel{\text { def }}{=}-\mathrm{e}^{R(x, t / \beta)} \mathrm{e}^{-\phi(x, t)}, \\
& \phi(x, t) \stackrel{\text { def }}{=} S(x, t / \beta) / \beta, \\
& B \stackrel{\text { def }}{=}-\gamma / \beta^{2}=-\gamma /(\delta-1)>0 .
\end{aligned}
$$

In the present case of (10) therefore

$$
\begin{aligned}
r(x, t) & =[\rho(x, t / \beta) /(-2 \gamma)]^{\frac{1}{2}} \mathrm{e}^{\phi(x, t)} \\
& =\left[\frac{\alpha_{1}+4 a^{2} \beta^{2} d n^{2}(a(x-v t), \kappa)}{-2 \gamma}\right]^{1 / 2} \mathrm{e}^{\phi(x, t)},
\end{aligned}
$$




$$
\begin{aligned}
s(x, t) & =-[\rho(x, t / \beta) /(-2 \gamma)]^{\frac{1}{2}} \mathrm{e}^{-\phi(x, t)} \\
& =-\left[\frac{\alpha_{1}+4 a^{2} \beta^{2} d n^{2}(a(x-v t), \kappa)}{-2 \gamma}\right]^{1 / 2} \mathrm{e}^{-\phi(x, t)} .
\end{aligned}
$$

As before, we see in (11) that $S$ is a potential function for the velocity field $u$. Note also that by (3) and (11) we obtain the solution

$$
\begin{aligned}
\psi(x, t) & =[\rho(x, t) /(-2 \gamma)]^{\frac{1}{2}} \mathrm{e}^{-i S(x, t)} \\
& =\left[\frac{\alpha_{1}+4 a^{2} \beta^{2} d n^{2}(a(x-\beta v t), \kappa)}{-2 \gamma}\right]^{1 / 2} \mathrm{e}^{-i S(x, t)},
\end{aligned}
$$

of the RNLS equation (1).

The formulas (11), (14), (15) relate the nonlinear systems (4), (12) and the resonance nonlinear Schrödinger Equation (1) to the cold plasma system (5) with solution (10). In Section 2 we relate the solution (10) also to nonlinear systems of Broer-Kaup, Boussinesq, and Hamilton-Jacobi-Bellman and their anti-systems given by the time reversal $t \rightarrow-t$. Here we find solutions of these systems that generalized in a non-trivial way those found in [11], for example. By choosing $\kappa=1$ so that $d n(x, \kappa) \stackrel{\text { def }}{=} \operatorname{sech}(x)$, and choosing $\alpha_{1}=0$ so that $C=0$ by (9) and $u=u_{0}$ is a constant function in (10), in particular, our $r$ in (14) and $v^{+}$in (34) of section 2 reduce to the dissipaton $e^{+}$and shock soliton $v^{+}$in Sections 2 and 4 of [11].

Attention in Section 4 is turned to further remarks on the cold plasma-2d black hole connection set up in [10]. The main result of that section is the computation of two more plasma dilaton fields such that these combined with the one computed in [10] form a linearly independent set. This too generalizes in a non-trivial way (namely the case $\alpha_{1} \neq 0$ ) a result found in [12].

Finally, in Section 5, we switch from the traveling wave solution $(\rho, u)$ to a plane wave solution of the system (5). Remarkably, its dispersion relation coincides with the dispersion relation obtained from the linearization of the RNLS Equation (1) about a suitably normalized ground state solution $\psi_{0}$.

Throughout, an attempt is made to maintain an expository style in the presentation of the material, for the sake of completeness.

\section{Elliptic Function Solutions of Broer-Kaup, Boussinesq, and Hamilton-Jacobi-Bellman Systems}

In addition to the solutions (11), (14) of the Madelung fluid and RD systems (4), (12), and the RNLS solution (15) of (1), all of which were constructed by way of the G-K solution (10), we consider now solutions of nonlinear systems of Broer-Kaup (B-K), Boussinesq, and Hamilton-Jacobi-Bellman (H-J-B), and of their time reversal $(t \rightarrow-t)$ systems. Here, again, the G-K solution (10) of the MAS (5) plays an underlying, subtextual role.

\subsection{Conservation Laws and the B-K System}

From the RDS (12), conservation laws can be deduced by which, in turn, one can 
derive the B-K system. We provide a sketch of this derivation, for the sake of completeness, and we present a general elliptic function solution.

The continuity equation $\rho_{t}+(\rho u)_{x}=0$, or conservation law, in (5) is one of many such laws. The RDS (12) gives rise to the conservation law

$$
(r s)_{t}-\left(r_{x} s-r s_{x}\right)_{x}=0 \text {, }
$$

for example, for which the particular value $B=-\gamma / \beta^{2}$ there does not matter. To check (16), start with the equation $-\left(r_{x} s-r s_{x}\right)_{x} \stackrel{(i)}{=} r s_{x x}-s r_{x x}$. Multiply the first RD equation in (12) by $s$ and the second one by $r$. Addition of these two multiplied equations eliminates $B$ and gives the equation $0=r_{t} s+s_{t} r-r_{x x} s+s_{x x} r=(r s)_{t}-\left(r_{x} s-r s_{x}\right)_{x}$, by (i), which is the assertion in (16). In addition to (16), we also need the conservation law

$$
\left[r_{x} s_{x}+\frac{B}{2}(r s)^{2}\right]_{t}=\left[r_{x} s_{t}+s_{x} r_{t}\right]_{x}
$$

which also follows from (12). Namely, the 1.h.s. in (17) is $r_{x} s_{x t}+r_{x t} s_{x}+B r s\left[r s_{t}+r_{t} s\right]$, where the third term here is $\left(B r^{2} s\right) s_{t}+\left(B r s^{2}\right) r_{t}=\left(-r_{t}+r_{x x}\right) s_{t}+\left(s_{t}+s_{x x}\right) r_{t} \quad$ (by (12)) $=r_{x x} s_{t}+s_{x x} r_{t}$. Thus the 1.h.s. in (17) is $r_{x} s_{x t}+r_{x t} s_{x}+r_{x x} s_{t}+s_{x x} r_{t}=\left(r_{x} s_{t}\right)_{x}+\left(s_{x} r_{t}\right)_{x}$, by the equality of mixed partials: $r_{t x}=r_{x t}$ and $s_{t x}=s_{x t}$. Equation (17) is now established.

Define

$$
\sigma \stackrel{\text { def }}{=}-r s \text {. }
$$

For $r>0$ and $s<0$, we see that $\sigma(\log r)_{x} \stackrel{\text { def }}{=}-\frac{r s r_{x}}{r} \stackrel{(i i)}{=}-s r_{x}$ and similarly $\sigma(\log -s)_{x}=-r s s_{x} / s \stackrel{(i i i)}{=}-r s_{x}$, which says that the conservation law (16) can be written as

$$
\sigma_{t}\left(\stackrel{\text { def }}{=}-(r s)_{t}\right)=\left[\sigma(\log r)_{x}-\sigma(\log -s)_{x}\right]_{x} .
$$

Also by (ii), (iii), $\sigma_{x}=\sigma(\log r)_{x}+\sigma(\log -s)_{x} \Rightarrow$

$$
\sigma_{x x}=\left[\sigma(\log r)_{x}+\sigma(\log -s)_{x}\right]_{x},
$$

so that (19) and (20) together give the equations

$$
\sigma_{t}+\sigma_{x x}=\left[2 \sigma(\log r)_{x}\right]_{x}, \quad \sigma_{t}-\sigma_{x x}=\left[-2 \sigma(\log -s)_{x}\right]_{x},
$$

which suggests that one defines

$$
\begin{aligned}
& v^{+} \stackrel{\text { def }}{=}(\log r)_{x}=\frac{r_{x}}{r}, \quad v^{- \text {def }}=(\log -s)_{x}=\frac{s_{x}}{s}: \\
& \sigma_{t}+\sigma_{x x}=\left(2 \sigma v^{+}\right)_{x}, \quad \sigma_{t}-\sigma_{x x}=\left(-2 \sigma v^{-}\right)_{x} .
\end{aligned}
$$

Then

$\left(v^{+}\right)_{x}+\left(v^{+}\right)^{2}=\left(r_{x} / r\right)_{x}+r_{x}^{2} / r^{2}=\left(r r_{x x}-r_{x}^{2}\right) / r^{2}+r_{x}^{2} / r^{2}=r_{x x} / r=\left(r_{t}+B r^{2} s\right) / r$

(by (12)) $\stackrel{\text { def }}{=} r_{t} / r-B \sigma$ (by (18)) $=(\log r)_{t}-B \sigma$. That is, 


$$
\left[v_{x}^{+}+\left(v^{+}\right)^{2}+B \sigma\right]_{x}=(\log r)_{t x}=(\log r)_{x t} \stackrel{\text { def }}{=}\left(v^{+}\right)_{t} .
$$

Similarly, by (12) and (18) again,

$$
\begin{aligned}
\left(v^{-}\right)_{x}+\left(v^{-}\right)^{2}=s_{x x} / s=\left(-s_{t}+B r s^{2}\right) / s=-(\log -s)_{t}-B \sigma \Rightarrow \\
\quad\left[\left(v^{-}\right)_{x}+\left(v^{-}\right)^{2}+B \sigma\right]_{x}=-(\log -s)_{t x}=-(\log -s)_{x t} \stackrel{\text { def }}{=}-\left(v^{-}\right)_{t} .
\end{aligned}
$$

Putting the pieces together, (Equations (23)-(25)), we have derived from the RDS (12), for $r>0, s<0$, and $B$ arbitrary (not necessarily the specific value $B=-\gamma / \beta^{2}$ there) the Broer-Kaup systems

$$
\begin{aligned}
& \sigma_{t}+\sigma_{x x}=\left(2 \sigma v^{+}\right)_{x},\left(v^{+}\right)_{t}=\left[\left(v^{+}\right)_{x}+\left(v^{+}\right)^{2}+B \sigma\right]_{x} \\
& \sigma_{t}-\sigma_{x x}=-\left(2 \sigma v^{-}\right)_{x},\left(v^{-}\right)_{t}=-\left[\left(v^{-}\right)_{x}+\left(v^{-}\right)^{2}+B \sigma\right]_{x}
\end{aligned}
$$

for $\sigma$ and $v^{ \pm}$defined in (18) and (22). The second system in (26) (with the minus signs) corresponds to the time reversal $t \rightarrow-t$.

We can obtain elliptic function solutions of the systems in (26) immediately, by way of the solutions $r, s$ in (14). Note first that by (10), (14), (18)

$$
\begin{aligned}
\sigma(x, t) & \stackrel{\text { def }}{=}-r(x, t) s(x, t)=\rho(x, t / \beta) /(-2 \gamma) \\
& =\left[\alpha_{1}+4 a^{2} \beta^{2} d n^{2}(a(x-v t), \kappa)\right] /(-2 \gamma)
\end{aligned}
$$

Also by (14)

$$
\begin{aligned}
& \log r(x, t)=\frac{1}{2}[\log \rho(x, t / \beta)-\log (-2 \gamma)]+\phi(x, t) \\
& \log -s(x, t)=\frac{1}{2}[\log \rho(x, t / \beta)-\log (-2 \gamma)]-\phi(x, t) .
\end{aligned}
$$

From [9], the identities and differentiation formulas

$$
\begin{aligned}
& \operatorname{sn}^{2}(x, \kappa)+\operatorname{cn}^{2}(x, \kappa)=1, d n^{2}(x, \kappa)+\kappa^{2} \operatorname{sn}^{2}(x, \kappa)=1, \\
& \operatorname{sn}(x, 1)=\tanh (x), \operatorname{cn}(x, 1)=d n(x, 1)=\operatorname{sech}(x), \\
& \frac{\mathrm{d}}{\mathrm{d} x} \operatorname{sn}(x, \kappa)=\operatorname{cn}(x, \kappa) d n(x, \kappa), \frac{\mathrm{d}}{\mathrm{d} x} \operatorname{cn}(x, \kappa)=-\operatorname{sn}(x, \kappa) d n(x, \kappa), \\
& \frac{\mathrm{d}}{\mathrm{d} x} d n(x, \kappa)=-\kappa^{2} \operatorname{sn}(x, \kappa) \operatorname{cn}(x, \kappa)
\end{aligned}
$$

hold for the standard Jacobi elliptic functions $\operatorname{sn}(x, \kappa), c n(x, \kappa), d n(x, \kappa)$. The following is a useful inequality:

$$
\frac{s n^{2}(x, \kappa) c n^{2}(x, \kappa)}{d n^{2}(x, \kappa)} \leq 1 .
$$

By definitions (13), (11), (10), (9), respectively

$$
\begin{aligned}
\phi_{x}(x, t) & =\frac{S_{x}(x, t / \beta)}{\beta}=-\frac{u(x, t / \beta)}{2 \beta}=-\frac{u_{0}}{2 \beta}-\frac{C}{2 \beta \rho(x, t / \beta)} \\
& =-\frac{v}{2}-\frac{C}{2 \beta \rho(x, t / \beta)} .
\end{aligned}
$$


By (10) (again)

$$
\rho(x, t / \beta)=\alpha_{1}+4 a^{2} \beta^{2} d n^{2}(a(x-v t), \kappa)
$$

so that the last differentiation formula in (29) gives

$$
\rho_{x}(x, t / \beta)=-8 a^{3} \beta^{2} \kappa^{2}(\text { sncndn })(a(x-v t), \kappa),
$$

which with (22), (28), (31) and (32) gives

$$
\begin{aligned}
& v^{+}(x, t) \stackrel{\text { def }}{=}(\log r)_{x}(x, t)=\frac{-4 a^{3} \beta^{2} \kappa^{2}(\operatorname{sncndn})(a(x-v t), \kappa)-C / 2 \beta}{\rho(x, t / \beta)=\alpha_{1}+4 a^{2} \beta^{2} d n^{2}(a(x-v t), \kappa)}-\frac{v}{2}, \\
& v^{-}(x, t) \stackrel{\text { def }}{=}(\log -s)_{x}(x, t)=\frac{-4 a^{3} \beta^{2} \kappa^{2}(\operatorname{sncndn})(a(x-v t), \kappa)+C / 2 \beta}{\rho(x, t / \beta)=\alpha_{1}+4 a^{2} \beta^{2} d n^{2}(a(x-v t), \kappa)}+\frac{v}{2},
\end{aligned}
$$

for $C$ given in (9).

The formulas (27) and (34) therefore provide for a solution $\left(\sigma, v^{+}, v^{-}\right)$of the Broer-Kaup systems in (26). There we take $B=-\gamma / \beta^{2}$, since we used the specific solution $(r, s)$ in (14) of the RDS (12).

The solution $\left(\sigma, v^{+}\right)$of the first system in (26) vastly generalizes the one $\left(\rho, v^{+}\right)$found in [11], where the notation $\rho$ there corresponds to $\sigma$ here, and where a solution of the second system in (26) is not addressed.

The generalization here is not simply that of elliptic functions over hyperbolic function, but it is in large part due to the freedom to allow $\alpha_{1}$ to be non-zero: $\alpha_{1}>0$. Indeed for $\alpha_{1}=0$, all results of this paper, and those of [10], simplify greatly-mainly because then $C=0$, by (9). For $\alpha_{1}=0$, the formulas in (27) and (34), for example, reduce to

$$
\begin{aligned}
& \sigma(x, t)=4 a^{2} \beta^{2} d n^{2}(a(x-v t), \kappa) /(-2 \gamma), \\
& v^{ \pm}(x, t)=-a \kappa^{2} \frac{s n c n}{d n}(a(x-v t), \kappa) \mp \frac{v}{2},
\end{aligned}
$$

which, moreover, for $\kappa=1$ reduce further to

$$
\begin{aligned}
& \sigma(x, t)=4 a^{2} \beta^{2} \operatorname{sech}^{2}(a(x-v t)) /(-2 \gamma), \\
& v^{ \pm}(x, t)=-a \tanh (a(x-v t)) \mp \frac{v}{2},
\end{aligned}
$$

which apart from $v^{-}$are the results that appear in [11], with a different choice of constants-the $v^{+}$in (36) being the shock soliton mentioned in the introduction. We shall see in Section 3 that in case $\alpha_{1}=0$, there is also a nice choice for the potential function $S(x, t)$ in (11), and thus a nice expression for $\phi(x, t)$ in (13), (14), and for $\psi(x, t)$ in (15) exists in this case.

\subsection{Boussinesq Systems}

Given the bulk of details and formulas in Section 2.1, we can glide more easily through this section. A good number of equations for various mathematical models are referred to as Boussinesq equations. It is perhaps best then to consider the systems discussed here more properly as Boussinesq type systems. This prolific French researcher, Joseph Valentin Boussinesq (1842-1929) made numerous high level contributions to fluid mechanics that involved the theory of 
solitary waves and the KdV equation, for example, and contributions to several other fields including the propagation of light and the theory of linear elasticity.

Define the "pressure" functions $p^{ \pm}(x, t)$ by

$$
p^{ \pm} \stackrel{\text { def }}{=} B \sigma+\left(v^{ \pm}\right)_{x} .
$$

As before we will choose $B \stackrel{\text { def }}{=}-\gamma / \beta^{2}$. By (26),

$$
\left(v^{ \pm}\right)_{t}= \pm\left[\left(v^{ \pm}\right)_{x}+\left(v^{ \pm}\right)^{2}+B \sigma\right]_{x}= \pm\left[\left(v^{ \pm}\right)_{x x}+2 v^{ \pm}\left(v^{ \pm}\right)_{x}+B \sigma_{x}\right]
$$

so that

$$
\left(v^{ \pm}\right)_{t} \mp 2 v^{ \pm}\left(v^{ \pm}\right)_{x}= \pm\left(v^{ \pm}\right)_{x x} \pm B \sigma_{x} \stackrel{\text { def }}{=} \pm\left(p^{ \pm}\right)_{x} .
$$

One can compute $\left(p^{ \pm}\right)_{t}$ also, and in the end, with (39), derive the Boussinesq type systems

$$
\begin{aligned}
& \left(p^{+}\right)_{x}=\left(v^{+}\right)_{t}-2 v^{+}\left(v^{+}\right)_{x},\left(p^{+}\right)_{t}=\left(v^{+}\right)_{x x x}+\left(2 p^{+} v^{+}\right)_{x} \\
& -\left(p^{-}\right)_{x}=\left(v^{-}\right)_{t}+2 v^{-}\left(v^{-}\right)_{x},\left(p^{-}\right)_{t}=-\left(v^{-}\right)_{x x x}-\left(2 p^{-} v^{-}\right)_{x}
\end{aligned}
$$

The second system in (40) (with the minus signs) corresponds to the time reversal $t \rightarrow-t$

As with the B-K systems in (26), one can obtain explicit elliptic function solutions of the systems in (40), or simpler hyperbolic function solutions as in (36), by taking $\kappa=1$. For this one simply applies the formulas for $\sigma$ and $v^{ \pm}$in (27) and (34) to compute $p^{ \pm}$in definition (37) - perhaps with the help of Maple. Generality in an alternate direction of importance is provided by Professor Pashaev in [11] who considers kink-soliton and two-soliton solutions of the Broer-Kaup and Boussinesq systems (26) and (40). These latter systems trace back to the analysis of water waves propagating in a long narrow channel [13] [14].

\subsection{A Hamilton-Jacobi-Bellman System}

Given two functions $f_{1}(x, t), f_{2}(x, t)$, consider the Lagrangian density

$$
\mathcal{L} \stackrel{\text { def }}{=} \frac{f_{2}}{2}\left[\frac{\partial f_{1}}{\partial t}-\frac{\partial^{2} f_{1}}{\partial x^{2}}\right]-\frac{f_{2}}{4}\left(\frac{\partial f_{1}}{\partial x}\right)^{2}-\frac{B}{2} f_{2}^{2} .
$$

The corresponding Euler-Lagrange equations of motion are

$$
\frac{\partial}{\partial t}\left(\frac{\partial \mathcal{L}}{\partial f_{j t}}\right)+\frac{\partial}{\partial x}\left(\frac{\partial \mathcal{L}}{\partial f_{j x}}\right)-\frac{\partial^{2}}{\partial x^{2}}\left(\frac{\partial \mathcal{L}}{\partial f_{j x x}}\right)=\frac{\partial \mathcal{L}}{\partial f_{j}}
$$

for $j=1,2$, which are immediately computed:

$$
\begin{aligned}
& \frac{\partial \mathcal{L}}{\partial f_{1 t}}=\frac{f_{2}}{2}, \frac{\partial \mathcal{L}}{\partial f_{2 t}}=0, \frac{\partial \mathcal{L}}{\partial f_{1 x}}=-\frac{f_{2}}{2} \frac{\partial f_{1}}{\partial x}, \\
& \frac{\partial \mathcal{L}}{\partial f_{2 x}}=0, \frac{\partial \mathcal{L}}{\partial f_{1 x x}}=-\frac{f_{2}}{2}, \frac{\partial \mathcal{L}}{\partial f_{2 x x}}=0, \frac{\partial \mathcal{L}}{\partial f_{1}}=0, \\
& \frac{\partial \mathcal{L}}{\partial f_{2}}=\frac{1}{2}\left[\frac{\partial f_{1}}{\partial t}-\frac{\partial^{2} f_{1}}{\partial x^{2}}\right]-\frac{1}{4}\left(\frac{\partial f_{1}}{\partial x}\right)^{2}-B f_{2}
\end{aligned}
$$


so that the equations of motion are

$$
\begin{aligned}
& \frac{\partial f_{2}}{\partial t}-\left(f_{2} \frac{\partial f_{1}}{\partial x}\right)_{x}+\frac{\partial^{2} f_{2}}{\partial x^{2}}=0, \\
& \frac{\partial f_{1}}{\partial t}-\frac{\partial^{2} f_{1}}{\partial x^{2}}-\frac{1}{2}\left(\frac{\partial f_{1}}{\partial x}\right)^{2}-2 B f_{2}=0 .
\end{aligned}
$$

Now going back to the first B-K equation

$$
\sigma_{t}-\left(\sigma 2 v^{+}\right)_{x}+\sigma_{x x}=0
$$

in (26), which we compare with the first equation in (44), we are obviously motivated to think of $f_{2}$ as $\sigma$ and to take

$$
\frac{\partial f_{1}}{\partial x}=2 v^{+} \stackrel{\text { def }}{=}(2 \log r)_{x} \text {. }
$$

That is, we choose

$$
f_{1}=A^{+} \stackrel{\text { def }}{=} 2 \log r
$$

Then (44) is the system

$$
\begin{aligned}
& \sigma_{t}-\left[\sigma\left(A^{+}\right)_{x}\right]_{x}+\sigma_{x x}=0 \\
& \left(A^{+}\right)_{t}-\left(A^{+}\right)_{x x}-\frac{1}{2}\left[\left(A^{+}\right)_{x}\right]^{2}-2 B \sigma=0
\end{aligned}
$$

where the second equation in (48) is a Hamilton-Jacobi-Bellman (H-J-B) equation, which as remarked in [11] is well-known in the theory of optimal stochastic control for continuous Markov processes [15]. Although this equation was derived by way of a suitable action functional, defined by the integration of $\mathcal{L}$ in (41) for choices $f_{1}=2 \log r\left(\stackrel{\text { def }}{=} A^{+}\right), f_{2}=\sigma$, another path to it is by way of the first $\mathrm{RD}$ equation in (12) divided by $r$;

$$
\begin{aligned}
0 & =\frac{r_{t}}{r}-\frac{r_{x x}}{r}+B r s \stackrel{\text { def }}{=}(\log r)_{t}-\frac{r_{x x}}{r}-B \sigma \\
& =\left(\frac{A^{+}}{2}\right)_{t}-\left[\left(v^{+}\right)_{x}+\left(v^{+}\right)^{2}\right]-B \sigma
\end{aligned}
$$

where the bracketed expression here for $r_{x x} / r$ was obtained in the first sentence that followed Equation (23). By the definition (22), and the definition of $A^{+}$again, $v^{+}=\left(A^{+} / 2\right)_{X} .(49)$ is therefore the statement

$$
0=\frac{1}{2}\left(A^{+}\right)_{t}-\frac{1}{2}\left(A^{+}\right)_{x x}-\frac{1}{4}\left[\left(A^{+}\right)_{x}\right]^{2}-B \sigma
$$

which is the H-J-B equation in (48).

At hand already we have a solution $\left(\sigma, A^{+}\right)$of the H-J-B system (48), by formulas (27) and (28). (27) gives $\sigma(x, t)$, and we can plug the expression for $\rho(x, t / \beta)$ there into the first formula in (28) to get

$$
\begin{aligned}
A^{+}(x, t) & \stackrel{\text { def }}{=} 2 \log r(x, t) \\
& =\log \left[\alpha_{1}+4 a^{2} \beta^{2} d n^{2}(a(x-v t), \kappa)\right]-\log (-2 \gamma)+2 \phi(x, t) \\
& =\log \sigma(x, t)+2 \phi(x, t)
\end{aligned}
$$


with $\phi_{x}$ given in (31). Again we choose $B=-\gamma / \beta^{2}$.

The useful role of the reaction diffusion system (12) has been noted several times in this section. Typically in physics, chemistry, or biology, for example, a more general system of the form

$$
\begin{aligned}
& r_{t}-d_{r} r_{x x}+F(r, s)=0 \\
& s_{t}-d_{s} s_{x x}+G(r, s)=0
\end{aligned}
$$

is encountered, where $d_{r}, d_{s}$ are diffusion constants for $r, s$ and where $F(u, v), G(u, v)$ are growth and interaction functions. $r$ and $s$ could be concentration functions for two chemicals, or prey and predator functions in a two-species ecological model, for example.

\section{Further Remarks on the Case $\alpha_{1}=0$}

It was pointed out in Section 2.1 that various formulas presented in this paper (and in the paper [10]) simplify drastically in case $\alpha_{1}=0$; in general $\alpha_{1} \geq 0$. Here we find in this case, in particular, a concrete expression for the potential function $S(x, t)$ (and thus also for the function $\phi(x, t))$ appearing in some of these formulas.

For $\alpha_{1}=0, u(x, t)=u_{0}=\beta v$, by (9) and (10), is a constant function: $C=0$. By (11), $S_{x}=-\beta v / 2, \gamma \mathrm{e} \stackrel{2 R}{=} \stackrel{\text { def }}{=}-\rho / 2$, and

$$
R_{x x}+R_{x}^{2}=\frac{1}{2}\left[\frac{\rho_{x x}}{2}-\frac{1}{2}\left(\frac{\rho_{x}}{\rho}\right)^{2}\right]
$$

by straight-forward differentiation of $R$. By Maple, for example, the differentiation of $\rho$ on the r.h.s. of (53) can be carried out. For $\rho$ given by (10) and $w \stackrel{\text { def }}{=} a(x-\beta t)$, the result is that this r.h.s. is $a^{2} \kappa^{2}\left[2 \operatorname{sn}^{2}(w, \kappa)-1\right]$, or $a^{2}\left[-2 d n^{2}(w, \kappa)+2-\kappa^{2}\right]$ by (29). As we have chosen $\delta$ in (1) to be $\beta^{2}+1$, we see that the second fluid equation in (4) therefore gives

$$
\begin{aligned}
0 & =S_{t}-\beta^{2} a^{2}\left[-2 d n^{2}(w, \kappa)+2-\kappa^{2}\right]-\frac{\beta^{2} v^{2}}{4}-\frac{\rho}{2} \\
& =S_{t}-\beta^{2} a^{2}\left(2-\kappa^{2}\right)-\frac{\beta^{2} v^{2}}{4}
\end{aligned}
$$

since $\rho / 2=2 a^{2} \beta^{2} d n^{2}(w, \kappa)$ by (10), for $\alpha_{1}=0$. That is, $S_{t}(x, t)$ is the constant function $\beta^{2} a^{2}\left(2-\kappa^{2}\right)+\beta^{2} v^{2} / 4$, or

$$
S(x, t)=\left[\beta^{2} a^{2}\left(2-\kappa^{2}\right)+\frac{\beta^{2} v^{2}}{4}\right] t+f(x),
$$

for a function of integration $f(x)$. Using again that $S_{x}=-\beta v / 2$, we see that $f^{\prime}(x)=-\beta v / 2 \Rightarrow f(x)=-\beta v x / 2+c_{0}$, for some constant $c_{0}$. Thus, in the end, for $\alpha_{1}=0$ we can choose the potential function $S(x, t)$ and the associated function $\phi(x, t)$ in (13) to be given by

$$
\begin{aligned}
& S(x, t)=\beta^{2}\left[a^{2}\left(2-\kappa^{2}\right)+\frac{v^{2}}{4}\right] t-\frac{\beta v x}{2}+c_{0}, \\
& \phi(x, t) \stackrel{\text { def }}{=} S(x, t / \beta) / \beta=\left[a^{2}\left(2-\kappa^{2}\right)+\frac{v^{2}}{4}\right] t-\frac{v x}{2}+\frac{c_{0}}{\beta}
\end{aligned}
$$


for a constant $c_{0}$.

These expressions for $S(x, t)$ and $\phi(x, t)$ can be plugged into formulas (14), (15), and (51), for example-taking $\alpha_{1}=0$ there, to further explicate the solutions $r, s, \psi, A^{+}$; keep in mind the assumption $\gamma<0$ :

$$
\begin{aligned}
& r(x, t)=\frac{2 a \beta}{\sqrt{-2 \gamma}} d n(a(x-v t), \kappa) \mathrm{e}^{\phi(x, t)} \\
& s(x, t)=\frac{-2 a \beta}{\sqrt{-2 \gamma}} d n(a(x-v t), \kappa) \mathrm{e}^{-\phi(x, t)} \\
& \psi(x, t)=\frac{2 a \beta}{\sqrt{-2 \gamma}} d n(a(x-v t), \kappa) \mathrm{e}^{-i S(x, t)} \\
& A^{+}(x, t)=\log \left(\frac{\left[4 a^{2} \beta^{2} d n^{2}(a(x-v t), \kappa)\right]}{-2 \gamma}\right)+2 \phi(x, t)
\end{aligned}
$$

for $\phi(x, t), S(x, t)$ given in (56). We also have the formulas for $\sigma(x, t)$ and $v^{ \pm}(x, t)$ in (35) in case $\alpha_{1}=0 . \operatorname{In}(57), 2 \beta / \sqrt{-2 \gamma}=\sqrt{2 / B}$, by (13).

Some concluding remarks about the case $\alpha_{1}=0$ pertain to the conservation laws (16) and (17). These laws imply that $r s$ and $r_{x} s_{x}+B(r s)^{2} / 2$ are conserved quantities that give rise to constants of motion

$$
I \stackrel{\text { def }}{=} \int_{-\infty}^{\infty} r s \mathrm{~d} x, \quad J \stackrel{\text { def }}{=} \int_{-\infty}^{\infty}\left[r_{x} s_{x}+B(r s)^{2} / 2\right] \mathrm{d} x .
$$

In [16], for example, where $\alpha_{1}=0, \kappa=1$ (and $\beta=1$ ), the mass $M \stackrel{\text { def }}{=}-I$ and energy $E \stackrel{\text { def }}{=} 2 J$ constants, in particular, are computed, where the notation $e^{+}, e^{-}$there (as in [5] [6] [11]) corresponds to $r, s$ here; also $-\Lambda / 4$ there corresponds to $B \stackrel{\text { def }}{=}-\gamma / \beta^{2}$ in (13) here. We state the results in terms of our notation, but we also express $M$ and $E$ in terms of the Gurevich-Krylov solution $\rho(x, t)$ in (10). First note that by (10), (29), (33), (36)

$$
\begin{aligned}
{\left[\frac{\rho_{x}}{\rho}(x, t / \beta)\right]^{2} } & =\left[\frac{-8 a^{3} \beta^{2} \tanh (a(x-v t)) \operatorname{sech}^{2}(a(x-v t))}{4 a^{2} \beta^{2} \operatorname{sech}^{2}(a(x-v t))}\right]^{2} \\
& =4 a^{2} \tanh ^{2}(a(x-v t))=4\left[\left(v^{+} v^{-}\right)(x, t)+\frac{v^{2}}{4}\right]
\end{aligned}
$$

for $\alpha_{1}=0, \kappa=1$. Then by (18), (22), (27), (59) $r_{x} s_{x}=r v^{+} s v^{-}=-\sigma v^{+} v^{-}$, $(r s)^{2}=\sigma^{2} \Rightarrow$

$$
\begin{aligned}
& {\left[r_{x} S_{x}+B(r s)^{2} / 2\right](x, t)=-\sigma(x, t)\left[v^{+} v^{-}-\frac{B}{2} \sigma\right](x, t)} \\
& =\frac{\rho(x, t / \beta)}{2 \gamma}\left[\frac{1}{4}\left(\frac{\rho_{x}}{\rho}\right)^{2}(x, t / \beta)-\frac{v^{2}}{4}-\frac{B}{2} \frac{\rho(x, t / \beta)}{-2 \gamma}\right] .
\end{aligned}
$$

The result is that

$$
\begin{aligned}
& M \stackrel{\text { def }}{=}-\int_{-\infty}^{\infty}(r s)(x, t) \mathrm{d} x \stackrel{\text { def }}{=} \int_{-\infty}^{\infty} \frac{\rho(x, t / \beta)}{-2 \gamma} \mathrm{d} x=\frac{4 a \beta^{2}}{-\gamma}, \\
& E \stackrel{\text { def }}{=} 2 \int_{-\infty}^{\infty}\left[r_{x} s_{x}-\frac{\gamma}{2 \beta^{2}}(r s)^{2}\right](x, t) \mathrm{d} x
\end{aligned}
$$




$$
\begin{aligned}
& =2 \int_{-\infty}^{\infty} \frac{\rho(x, t / \beta)}{-2 \gamma}\left(\frac{1}{4}\left(\frac{\rho_{x}}{\rho}\right)^{2}(x, t / \beta)-\frac{v^{2}}{4}\right)-\frac{\rho(x, t / \beta)^{2}}{8 \gamma \beta^{2}} \mathrm{~d} x \\
& =-\frac{8 a^{3} \beta^{2}}{3 \gamma}-\frac{2 a \beta^{2} v^{2}}{\gamma},
\end{aligned}
$$

using that

$$
\begin{aligned}
& \int_{-\infty}^{\infty} \operatorname{sech}^{2}(a(x-v t)) \mathrm{d} x=\frac{2}{a}, \quad \int_{-\infty}^{\infty} \operatorname{sech}^{4}(a(x-v t)) \mathrm{d} x=\frac{4}{3 a}, \\
& \int_{-\infty}^{\infty}\left(\tanh ^{2} \operatorname{sech}^{2}\right)(a(x-v t)) \mathrm{d} x=\frac{2}{3 a},
\end{aligned}
$$

where the latter integral formula in (62) is used to compute the integration of $r_{x} s_{x}$ in the definition of $E$. Namely, as we have just noted, $r_{x} s_{x}=-\sigma v^{+} v^{-}$so by (36) again

$$
\left(r_{x} s_{x}\right)(x, t)=\frac{4 a^{2} \beta^{2}}{2 \gamma} \operatorname{sech}^{2}(a(x-v t))\left[a^{2} \tanh ^{2}(a(x-v t))-\frac{v^{2}}{4}\right] .
$$

\section{Plasma Metric and Plasma Dilaton Fields}

An exact connection of the cold plasma system (5) to a two-dimensional Jackiw-Teitelboim (J-T) black hole was investigated in [10], with the resonance NLS Equation (1) serving as a bridge. An explicit change of variables was set up which transformed a plasma metric associated with the system (5) to a J-T metric. This transformation, moreover, provided for a direct calculation of a plasma dilaton field, which with the plasma metric and an appropriate cosmological constant constitutes a solution of the J-T gravitational field equations [17] [18]. The results of [10] represent an extension to the non-trivial case $\alpha_{1} \neq 0$ of results in [12] - a case discussed in Sections 2 and 3 here. As indicated in the introduction, two more plasma dilaton fields will be computed in this section, to obtain a set of three linearly independent ones altogether.

The J-T gravitational field equations are a system of equations

$$
\begin{aligned}
& R(g)+2 \Lambda=0 \\
& \nabla_{i} \nabla_{j} \Phi+\Lambda \Phi g_{i j}=0, \quad 1 \leq i, j \leq 2
\end{aligned}
$$

of which a solution consists of a triple $(g, \Phi, \Lambda)$ where $g$ is a pseudo Riemannian metric with local components $g_{i j}, R(g)$ is its Ricci scalar curvature, $\Phi$ is a real-valued function of the local coordinates $\left(x_{1}, x_{2}\right)$ in which $g$ is expressed (called a dilaton field), $\Lambda$ is a cosmological constant (and therefore the scalar curvature is a constant), and where, locally, the Hessian $\nabla_{i} \nabla_{j}$ is given by

$$
\nabla_{i} \nabla_{j} \Phi=\frac{\partial^{2} \Phi}{\partial x_{i} \partial x_{j}}-\sum_{k=1}^{2} \Gamma_{i j}^{k} \frac{\partial \Phi}{\partial x_{k}}
$$

for the Christoffel symbols $\Gamma_{i j}^{k}$ of $g$, of the second kind.

For example, the J-T (Lorentzian) black hole solution of the system (64) is given in the coordinates $\left(x_{1}, x_{2}\right)=(\tau, r)$ by $g$ with 


$$
\begin{aligned}
& \mathrm{d} s^{2} \stackrel{\text { def }}{=}-\left(m^{2} r^{2}-M\right) \mathrm{d} \tau^{2}+\frac{\mathrm{d} r^{2}}{m^{2} r^{2}-M}, \\
& \Phi(\tau, r) \stackrel{\text { def }}{=} m r, \Lambda \stackrel{\text { def }}{=}-m^{2}
\end{aligned}
$$

for fixed real numbers $m, M, M>0$ being a black hole mass parameter. Here $R(g)=2 m^{2}$, and we point out that the sign convention for scalar curvature that we adopt here (and in [10] [12]) is spelled out on page 182 of [19], for example. Thus, for example, our $R(g)$ is the negative of that employed in [17] [18]. We will also write $g_{b h}$ for the J-T metric given in (66).

As a second example, we consider the plasma metric $g=g_{\text {plasma }}$ constructed in [10] by way of the G-K solution in (10) of the MAS (5). Here the local coordinates $\left(x_{1}, x_{2}\right)=(\tau, \rho)$, with the notation $\rho$ here not to be confused with the same notation for the solution $\rho$ in (10):

$$
\begin{aligned}
g: \mathrm{d} s^{2} \stackrel{\text { def }}{=} A(\rho) \mathrm{d} \tau^{2}-\left[\frac{4 a^{4} \beta^{4} \kappa^{4}}{\gamma^{2} A(\rho)}\left(s n^{2} c n^{2} d n^{2}\right)(\rho, \kappa)\right] \mathrm{d} \rho^{2}, \\
A(\rho) \stackrel{\text { def }}{=} 4 a^{2} \beta^{2}\left[\frac{a^{2} \kappa^{4}}{-2 \gamma}\left(s n^{2} c n^{2}\right)(\rho, \kappa)+\frac{v^{2}}{8 \gamma} \mathrm{d} n^{2}(\rho, \kappa)\right] \\
+\frac{16 \alpha_{1} a^{4} \beta^{2} \kappa^{4}\left(s n^{2} c n^{2}\right)(\rho, \kappa)+C^{2} / \beta^{2}}{8 \gamma\left[\alpha_{1}+4 a^{2} \beta^{2} d n^{2}(\rho, \kappa)\right]}+\frac{\alpha_{1} v^{2}}{8 \gamma}-\frac{v C}{4 \gamma \beta}
\end{aligned}
$$

where (again) $C$ is given (9). Obviously this metric is more complicated in structure. For $\alpha_{1}=0$ (so that again $C=0$ ) and for the choice $\gamma=-1 / 2$ (as in [5] [6]) it reduces to the metric (6) in [12], where the notation $b$ there corresponds to $2 \beta$ here. Some remarks herewith are offered to provide some clarification regarding the "raisons d'être" of the plasma metric formula (67).

The classical continuous Heisenberg model realized on a single sheeted hyperboloid

$$
-S_{1}(x, t)^{2}+S_{2}(x, t)^{2}-S_{3}(x, t)^{2}=-1
$$

with equations of motion

$$
\mathcal{S}_{t}=\frac{1}{2 i}\left[\mathcal{S}, \mathcal{S}_{x x}\right], \quad \mathcal{S} \stackrel{\text { def }}{=} i\left[\begin{array}{cc}
S_{3} & S_{1}-S_{2} \\
S_{1}+S_{2} & -S_{3}
\end{array}\right]
$$

is equipped with a canonical metric

$$
\begin{aligned}
& g_{H}: \mathrm{d} s^{2}=g_{11} \mathrm{~d} t^{2}+2 g_{12} \mathrm{~d} t \mathrm{~d} x+g_{22} \mathrm{~d} x^{2} \\
& g_{11} \stackrel{\text { def }}{=}-\left(\frac{\partial S_{1}}{\partial t}\right)^{2}+\left(\frac{\partial S_{2}}{\partial t}\right)^{2}-\left(\frac{\partial S_{3}}{\partial t}\right)^{2} \\
& g_{22} \stackrel{\text { def }}{=}-\left(\frac{\partial S_{1}}{\partial x}\right)^{2}+\left(\frac{\partial S_{2}}{\partial x}\right)^{2}-\left(\frac{\partial S_{3}}{\partial x}\right)^{2} \\
& g_{12} \stackrel{\text { def }}{=}-\frac{\partial S_{1}}{\partial t} \frac{\partial S_{1}}{\partial x}+\frac{\partial S_{2}}{\partial t} \frac{\partial S_{2}}{\partial x}-\frac{\partial S_{3}}{\partial t} \frac{\partial S_{3}}{\partial x}
\end{aligned}
$$

of constant scalar curvature $R\left(g_{H}\right)=2$. The bracket in (69) is just the commutator $\left[M_{1}, M_{2}\right] \stackrel{\text { def }}{=} M_{1} M_{2}-M_{2} M_{1}$ of two matrices $M_{1}$ and $M_{2}$, and the $g_{i j}$ 
in (70) are simply Minkowski inner product expressions: $\langle X, Y\rangle \stackrel{\text { def }}{=}-x_{1} y_{1}+x_{2} y_{2}-x_{3} y_{3}$ for $X=\left(x_{1}, x_{2}, x_{3}\right), \quad Y=\left(y_{1}, y_{2}, y_{3}\right) \in \mathbb{R}^{3}$.

On the other hand, one can construct Lax pairs $\left(J_{0}(\lambda), J_{1}(\lambda)\right)$ for this Heisenberg model, and Lax pairs $\left(u_{0}(\lambda), u_{1}(\lambda)\right)$ for the reaction diffusion system (12), and establish a gauge equivalence of these two nonlinear systems [16]. More precisely, in our specific setup,

$$
\begin{aligned}
& J_{0}(\lambda) \stackrel{\text { def }}{=} \frac{i \lambda^{2} \mathcal{S}}{8}+\frac{\lambda}{4} \mathcal{S} \mathcal{S}_{x}, \quad J_{1}(\lambda) \stackrel{\text { def }}{=} \frac{i \lambda \mathcal{S}}{4} \\
& u_{0}(\lambda) \stackrel{\operatorname{def}}{=}\left[\begin{array}{ll}
\lambda^{2} / 8-\tilde{r} \tilde{s} & -\tilde{s}_{x}+\lambda \tilde{s} / 2 \\
\tilde{r}_{x}+\lambda \tilde{r} / 2 & -\lambda^{2} / 8+\tilde{r} \tilde{s}
\end{array}\right], \quad u_{1}(\lambda) \stackrel{\text { def }}{=}\left[\begin{array}{cc}
\lambda / 4 & \tilde{s} \\
\tilde{r} & -\lambda / 4
\end{array}\right] \\
& \tilde{r} \stackrel{\text { def }}{=} \sqrt{\frac{B}{2}} r, \quad \tilde{s} \stackrel{\text { def }}{=} \sqrt{\frac{B}{2}} s,
\end{aligned}
$$

for a (complex) spectral parameter $\lambda$. The equations of motion assertion (69) is equivalent to the assertion that $\left(J_{0}(\lambda), J_{1}(\lambda)\right)$ satisfies the zero curvature condition $(\mathrm{zcc})$

$$
J_{1}(\lambda)_{t}-J_{0}(\lambda)_{x}+\left[J_{0}(\lambda), J_{1}(\lambda)\right]=0
$$

Similarly, a pair of functions $(r(x, t), s(x, t))$ is a solution of the RDS (12) if and only if $\left(u_{0}(\lambda), u_{1}(\lambda)\right)$ satisfies the zcc

$$
u_{1}(\lambda)_{t}-u_{0}(\lambda)_{x}+\left[u_{0}(\lambda), u_{1}(\lambda)\right]=0
$$

Gauge equivalence means that for some $M \in G L(2, \mathbb{R})$,

$$
\begin{aligned}
& u_{0}^{M}(\lambda)=J_{0}(\lambda), \quad u_{1}^{M}(\lambda)=J_{1}(\lambda), \\
& u_{0}^{M}(\lambda) \stackrel{\text { def }}{=} M u_{0}(\lambda) M^{-1}-M_{t} M^{-1}, \\
& u_{1}^{M}(\lambda) \stackrel{\text { def }}{=} M u_{1}(\lambda) M^{-1}-M_{x} M^{-1},
\end{aligned}
$$

which in turn means that one can show that the Heisenberg metric $g_{H}$ in (70) can be expressed in terms of $r$ and $s$. The result (a key result) is that

$$
g_{11}=2 B\left(-r_{x} s_{x}\right), \quad g_{12}=B\left(r_{x} s-r s_{x}\right), \quad g_{22}=2 B r s .
$$

Since the scalar curvature $R\left(g_{H}\right)$ of $g_{H}$ has the value 2, as we have noted, it is convenient to work with the metric $g_{H} / 2 B$ with scalar curvature $4 B \stackrel{\text { def }}{=}-4 \gamma / \beta^{2}$. Now $r s=-\sigma$ by definition (18), so by (27)

$$
\left(g_{H} / 2 B\right)_{22}(x, t) \stackrel{\text { def }}{=}(r s)(x, t)=\left[\alpha_{1}+4 a^{2} \beta^{2} d n^{2}(a(x-v t), \kappa)\right] / 2 \gamma .
$$

By formulas (34) then

$$
\left(v^{+}-v^{-}\right)(x, t)=\frac{-C / \beta}{2 \gamma(r s)(x, t)}-v,
$$

and by definition (22), $r_{x} s-r s_{x}=v^{+} r s-v^{-} s r=\left(v^{+}-v^{-}\right) r s=-C / 2 \gamma \beta-v r s$ by (77). That is, by (75), (76)

$$
\left(g_{H} / 2 B\right)_{12}(x, t)=-\frac{C}{4 \gamma B}-\frac{v}{4 \gamma}\left[\alpha_{1}+4 a^{2} \beta^{2} d n^{2}(a(x-v t), \kappa)\right] .
$$


The computation of $\left(g_{H} / 2 B\right)_{11} \stackrel{\text { def }}{=}-r_{x} s_{x}$ (by (75)) is more involved. By (18) and (22) again, $-r_{X} s_{x}=\sigma v^{+} v^{-}$, which can be computed by (27) and (34) again. The end result is that

$$
\begin{aligned}
\left(g_{H} / 2 B\right)_{11}= & 4 a^{2} \beta^{2}\left[\frac{a^{2} \kappa^{4}}{-2 \gamma}\left(s n^{2} c n^{2}\right)(a(x-v t), \kappa)+\frac{v^{2}}{8 \gamma} d n^{2}(a(x-v t), \kappa)\right] \\
& +\frac{16 \alpha_{1} a^{4} \beta^{2} \kappa^{4}\left(s n^{2} c n^{2}\right)(a(x-v t), \kappa)+C^{2} / \beta^{2}}{8 \gamma\left[\alpha_{1}+4 a^{2} \beta^{2} d n^{2}(a(x-v t), \kappa)\right]}+\frac{\alpha_{1} v^{2}}{8 \gamma}+\frac{v C}{4 \gamma \beta} .
\end{aligned}
$$

In the very specialized case of $\alpha_{1}=0, \kappa=1,-\left(g_{H} / 2 B\right)_{11}$ is given exactly by Equation (63). Formulas (76), (78), (79) (for $C$ given in (9)), which give the structure of the metric $g_{H} / 2 B$ were also obtained in [10], by a different method. This metric is non-diagonal: $\left(g_{H} / 2 B\right)_{12}=\left(g_{H} / 2 B\right)_{21} \neq 0$. In Section 3 of [10] a suitable change of variables was constructed by which $g_{H} / 2 B$ was transformed exactly to the plasma metric $g$ in (67), which is diagonal. For this, $v^{2}$ is required to be sufficiently large:

$$
v^{2}>4 a^{2} \kappa^{4} \text { and } v^{2} \geq \frac{4 C^{2}}{\alpha_{1}^{2} \beta^{2}} \text { for } \alpha_{1}>0,
$$

with the single condition

$$
v^{2}>4 a^{2} \kappa^{4} \text { for } \alpha_{1}=0 .
$$

A clarification regarding how the plasma metric $g_{\text {plasma }}$ in (67) arises is now established. Also $R\left(g_{\text {plasma }}\right)=R\left(g_{H} / 2 B\right)=-4 \gamma / \beta^{2}$, as we have seen.

We move on now to the main result in [10], which will allow, in particular, for a direct computation of plasma dilaton fields and thus for solutions of the field Equations (64). That main result states that the change of variables $(\tau, \rho) \rightarrow(\tau, r=\psi(\rho))$ for

$$
\psi(\rho) \stackrel{\text { def }}{=} \frac{a^{2} \beta^{2} d n^{2}(\rho, \kappa)}{-\gamma}+\frac{a^{2} \beta^{2}}{2 \gamma}\left(2-\kappa^{2}\right)-\frac{\left(v^{2} \beta^{2}-\alpha_{1}\right)}{8 \gamma}
$$

transforms the cold plasma metric $g_{\text {plasma }}$ in (67) precisely to the J-T black hole metric $g_{b h}$ in (66), for $m$ and $M$ there given by

$$
\begin{aligned}
m \stackrel{\text { def }}{=} & +\frac{(-2 \gamma)^{1 / 2}}{\beta}, \\
M \stackrel{\text { def }}{=} & \frac{-v C}{4 \gamma \beta}+\frac{a^{2} \beta^{2} v^{2}}{2 \gamma}-\frac{a^{4} \beta^{2} \kappa^{4}}{2 \gamma}-\frac{a^{2} \beta^{2} \kappa^{2} v^{2}}{4 \gamma}-\frac{\beta^{2} v^{4}}{32 \gamma} \\
& +\frac{3 \alpha_{1} v^{2}}{16 \gamma}+\frac{3 \alpha_{1}^{2}}{32 \gamma \beta^{2}}-\frac{\alpha_{1} a^{2} \kappa^{2}}{4 \gamma}+\frac{\alpha_{1} a^{2}}{2 \gamma} .
\end{aligned}
$$

Another (more compact) expression is given in (99) for the black hole mass parameter $M$ here, which is indeed positive for

$$
v^{2}>8 a^{2}\left(2-\kappa^{2}\right)+\frac{6 \alpha_{1}}{\beta^{2}}, \quad v \geq \frac{\alpha_{1}}{C}\left[\frac{3 \alpha_{1}}{8 \beta}+2 a^{2} \beta\right],
$$

which we assume. Of course the second condition (84) is automatic for $\alpha_{1}=0$ 
and the first condition, in fact the condition $v^{2}>8 a^{2}\left(2-\kappa^{2}\right)$, implies the first condition $v^{2}>4 a^{2} \kappa^{4}$ in (80).

Going back to the earlier equations $R\left(g_{b h}\right)=2 m^{2}$ and $R\left(g_{\text {plasma }}\right)=-4 \gamma / \beta^{2}$, we conclude from the equality of these scalar curvatures that $m$ must be given by (83), and moreover that by the first field equation in (64) the cosmological constant $\Lambda$ must be given by

$$
\Lambda=\frac{-R\left(g_{\text {plasma }}\right)}{2}=\frac{2 \gamma}{\beta^{2}}=-m^{2} .
$$

$\gamma<0$ throughout this paper, and we see that the cosmological constant is negative. The dilaton field $\Phi_{\text {plasma }}^{(1)}$ for the second set of equations in (64), for the plasma metric, can be derived immediately from the J-T dilaton field $\Phi(\tau, r)=m r$ in (66):

$$
\begin{aligned}
\Phi_{\text {plasma }}^{(1)}(\tau, \rho) & \stackrel{\text { def }}{=} \Phi(\tau, \psi(\rho)) \stackrel{\text { def }}{=} m \psi(\rho) \\
& \stackrel{\text { def }}{=} m\left[\frac{a^{2} \beta^{2} d n^{2}(\rho, \kappa)}{-\gamma}+\frac{a^{2} \beta^{2}}{2 \gamma}\left(2-\kappa^{2}\right)-\frac{v^{2} \beta^{2}-\alpha_{1}}{8 \gamma}\right]
\end{aligned}
$$

as in [10]. There are, however, two other J-T dilaton fields $\Phi^{(j)}(\tau, r), j=2,3$, that are also solutions of the second set of equations in (64) for the metric $g_{b h}$, and therefore by way of the transformation $\Psi$ in (82) there are in addition plasma dilaton fields $\Phi_{\text {plasma }}^{(j)}(\tau, \rho), j=2,3$, for the plasma metric which were not computed in [10], which we compute here.

In fact, one can take

$$
\begin{aligned}
& \Phi^{(2)}(\tau, r) \stackrel{\text { def }}{=} \sqrt{m^{2} r^{2}-M} \sinh (m \sqrt{M} \tau), \\
& \Phi^{(3)}(\tau, r) \stackrel{\text { def }}{=} \sqrt{m^{2} r^{2}-M} \cosh (m \sqrt{M} \tau)
\end{aligned}
$$

so that (as in (86))

$$
\begin{aligned}
& \Phi_{\text {plasma }}^{(2)}(\tau, \rho) \stackrel{\text { def }}{=} \Phi^{(2)}(\tau, \psi(\rho)) \stackrel{\text { def }}{=} \sqrt{m^{2} \psi(\rho)^{2}-M} \sinh (m \sqrt{M} \tau), \\
& \Phi_{\text {plasma }}^{(3)}(\tau, \rho) \stackrel{\text { def }}{=} \Phi^{(3)}(\tau, \psi(\rho)) \stackrel{\text { def }}{=} \sqrt{m^{2} \psi(\rho)^{2}-M} \cosh (m \sqrt{M} \tau)
\end{aligned}
$$

which means that we need to find expressions for $m^{2} \psi(\rho)^{2}-M$ and $m \sqrt{M}$, given the definitions (82), (83). For convenience, write for now sn, $c n, d n$ for $\operatorname{sn}(\rho, \kappa), \operatorname{cn}(\rho, \kappa), d n(\rho, \kappa)$. Let

$$
\begin{aligned}
w(\rho)=w \stackrel{\text { def }}{=} & 16 a^{4} \beta^{4}+16 a^{4} \beta^{4} \kappa^{2} d n^{2}+4 a^{2} \beta^{4} v^{2} d n^{2}-16 a^{4} \beta^{4} \kappa^{2} \\
& +16 a^{4} \beta^{4} d n^{4}-32 a^{4} \beta^{4} d n^{2}
\end{aligned}
$$

Then, as is easily checked, $w$ has factorization $w=16 a^{2} \beta^{4} B(\rho)$ for

$$
B(\rho) \stackrel{\text { def }}{=} d n^{2}\left[\frac{a^{2}\left(1-\kappa^{2}\right)}{d n^{2}}+a^{2} \kappa^{2}-2 a^{2}+\frac{v^{2}}{4}+a^{2} d n^{2}\right],
$$

which, in turn, can be written as 


$$
B(\rho)=d n^{2}\left[\frac{v^{2}}{4}-a^{2} \kappa^{4} \frac{s n^{2} c n^{2}}{d n^{2}}\right] .
$$

The argument in [12], for example, leading up to Equation (55) there verifies this assertion. Therefore

$$
w=16 a^{2} \beta^{4} d n^{2}\left[\frac{v^{2}}{4}-a^{2} \kappa^{4} \frac{s n^{2} c n^{2}}{d n^{2}}\right] .
$$

On the other hand, given the definitions (82), (83), one has by Maple, for example, that

$$
\begin{aligned}
& m^{2} \psi(\rho)^{2}-M \\
& =\left[w+\alpha_{1}^{2}+\alpha_{1} \beta^{2} v^{2}+8 \alpha_{1} a^{2} \beta^{2}-4 \alpha_{1} a^{2} \beta^{2} \kappa^{2}-4 \alpha_{1} a^{2} \beta^{2} d n^{2}-2 v \beta C\right] /\left(-8 \gamma \beta^{2}\right)
\end{aligned}
$$

for $C$ (as usual) in (9). Using that $d n^{2}-1=-\kappa^{2} s n^{2}$ and $1-s n^{2}=c n^{2}$ (by (29)), one can write $8 \alpha_{1} a^{2} \beta^{2}-4 \alpha_{1} a^{2} \beta^{2} \kappa^{2}-4 \alpha_{1} a^{2} \beta^{2} d n^{2}=4 \alpha_{1} a^{2} \beta^{2}\left[1-\kappa^{2} c n^{2}\right]$. In the end,

$$
\begin{aligned}
& m^{2} \psi(\rho)^{2}-M \\
& =\left[w(\rho)+\alpha_{1}\left\{\alpha_{1}+\beta^{2}\left(v^{2}+4 a^{2}\left(1-\kappa^{2} c n^{2}(\rho, \kappa)\right)\right)\right\}-2 v \beta C\right] /\left(-8 \gamma \beta^{2}\right)
\end{aligned}
$$

for

$$
\begin{aligned}
& w(\rho)=w=16 a^{2} \beta^{4} d n^{2}(\rho, \kappa)\left[\frac{v^{2}}{4}-a^{2} \kappa^{4} \frac{s n^{2} c n^{2}}{d n^{2}}(\rho, \kappa)\right], \\
& C \stackrel{\text { def }}{=}+\left(\alpha_{1} \alpha_{2} \alpha_{3}\right)^{\frac{1}{2}}=\left[\alpha_{1}\left(4 a^{2} \beta^{2}\left(1-\kappa^{2}\right)+\alpha_{1}\right)\left(4 a^{2} \beta^{2}+\alpha_{1}\right)\right]^{1 / 2} .
\end{aligned}
$$

Next, note that in (83)

$$
\begin{aligned}
& \frac{a^{2} \beta^{2} v^{2}}{2 \gamma}-\frac{a^{4} \beta^{2} \kappa^{4}}{2 \gamma}-\frac{a^{2} \beta^{2} \kappa^{2} v^{2}}{4 \gamma}-\frac{\beta^{2} v^{4}}{32 \gamma} \\
& =\frac{-\beta^{2}}{2 \gamma}\left[\frac{-a^{2} v^{2}}{2}\left(2-\kappa^{2}\right)+a^{4} \kappa^{4}+\frac{v^{4}}{16}\right]=\frac{\beta^{2}}{-2 \gamma} A
\end{aligned}
$$

for the same constant $A$ in definition (11) of [12]:

$$
A \stackrel{\text { def }}{=}-\frac{a^{2} v^{2}}{2}\left(2-\kappa^{2}\right)+a^{4} \kappa^{4}+\frac{v^{4}}{16} \text {. }
$$

Also in (83)

$$
\frac{3 \alpha_{1} v^{2}}{16 \gamma}+\frac{3 \alpha_{1}^{2}}{32 \gamma \beta^{2}}-\frac{\alpha_{1} a^{2} \kappa^{2}}{4 \gamma}+\frac{\alpha_{1} a^{2}}{2 \gamma}=\frac{\alpha_{1} \beta^{2}}{-2 \gamma}\left[-\frac{3 v^{2}}{8 \beta^{2}}-\frac{3 \alpha_{1}}{16 \beta^{4}}-\frac{a^{2}\left(2-\kappa^{2}\right)}{2 \beta^{2}}\right] .
$$

It follows that $M$ can also be expressed as

$$
M=\frac{\beta^{2}}{-2 \gamma}\left[\frac{v C}{2 \beta^{3}}+A-\alpha_{1}\left(\frac{3 v^{2}}{8 \beta^{2}}+\frac{3 \alpha_{1}}{16 \beta^{4}}+\frac{a^{2}\left(2-\kappa^{2}\right)}{2 \beta^{2}}\right)\right],
$$

where $\beta^{2} /-2 \gamma \stackrel{\text { def }}{=} 1 / m^{2}$. Then 


$$
m \sqrt{M}=\left[\frac{v C}{2 \beta^{3}}+A-\alpha_{1}\left(\frac{3 v^{2}}{8 \beta^{2}}+\frac{3 \alpha_{1}}{16 \beta^{4}}+\frac{a^{2}\left(2-\kappa^{2}\right)}{2 \beta^{2}}\right)\right]^{1 / 2}
$$

for $C$ and $A$ in (95) and (97), respectfully. In summary, the formulas for $m^{2} \psi(\rho)^{2}-M$ and $m \sqrt{M}$ in (94) and (100) provide for the computation of the plasma dilaton fields $\Phi_{\text {plasma }}^{(j)}(\tau, \rho), j=2,3$, in (88) for the plasma metric $g_{\text {plasma }}$ in (67).

As usual, all formulas simplify considerably in case $\alpha_{1}=0$ :

$$
\begin{aligned}
& m^{2} \psi(\rho)^{2}-M=\frac{w(\rho)}{-8 \gamma \beta^{2}} \stackrel{\text { def }}{=} \frac{16 a^{2} \beta^{4}}{-8 \gamma \beta^{2}} d n^{2}(\rho, \kappa)\left[\frac{v^{2}}{4}-a^{2} \kappa^{4} \frac{s n^{2} c n^{2}}{d n^{2}}(\rho, \kappa)\right], \\
& m \sqrt{M}=\sqrt{A} \stackrel{\text { def }}{=}\left[\frac{-a^{2} v^{2}}{2}\left(2-\kappa^{2}\right)+a^{4} \kappa^{4}+\frac{v^{4}}{16}\right]^{1 / 2},
\end{aligned}
$$

by (94), (95), (97), and (100), which gives

$$
\begin{aligned}
& \Phi_{\text {plasma }}^{(1)}(\tau, \rho)=\frac{m \beta^{2}}{-2 \gamma}\left[2 a^{2} d n^{2}(\rho, \kappa)-a^{2}\left(2-\kappa^{2}\right)+\frac{v^{2}}{4}\right], \\
& \Phi_{\text {plasma }}^{(2)}(\tau, \rho)=\sqrt{\frac{2}{-\gamma}} a \beta d n(\rho, \kappa)\left[\frac{v^{2}}{4}-a^{2} \kappa^{4} \frac{s n^{2} c n^{2}}{d n^{2}}(\rho, \kappa)\right]^{1 / 2} \sinh (\sqrt{A} \tau), \\
& \Phi_{\text {plasma }}^{(3)}(\tau, \rho)=\sqrt{\frac{2}{-\gamma}} a \beta d n(\rho, \kappa)\left[\frac{v^{2}}{4}-a^{2} \kappa^{4} \frac{s n^{2} c n^{2}}{d n^{2}}(\rho, \kappa)\right]^{1 / 2} \cosh (\sqrt{A} \tau) .
\end{aligned}
$$

Apart from the constant factors $m \beta^{2} /-2 \gamma=1 / m$ and $(2 /-\gamma)^{1 / 2} a \beta$, the dilaton fields in (102) (obtained for $\alpha_{1}=0$ ) are exactly the ones computed in [12], by a different method; see Equation (10) and Equation (11) there.

\section{A Plane Wave Solution of the Magnetoacoustic System}

The traveling wave solution $(\rho, u)$ in (10) of the magnetoacoustic system (5) has been of central interest, of course. Equation (15), with $S_{x}=-u / 2$ as in (11), relates this solution to the solution $\psi$ of the resonance NLS equation (1). There is another instance, or another sense, in which solutions of (5) and (1) are related, of which some brief remarks are added in this section.

The bond between the system (5) and Equation (1) is illustrated further in [20], for example, where it is shown that they share a dispersion relation. More precisely, in place of the traveling wave solution $(\rho, u)$ in $(10)$, consider a plane wave solution $(\rho, u)$ of (5) of the form

$$
\begin{aligned}
& \rho(x, t) \stackrel{\text { def }}{=} \rho_{0}+A_{1} \mathrm{e}^{i(k x-\omega t)} \\
& u(x, t) \stackrel{\text { def }}{=} A_{2} \mathrm{e}^{i(k x-\omega t)}
\end{aligned}
$$

for constants $\rho_{0} \neq 0, A_{1}, A_{2}$, wave frequency $\omega$, and wave number $k$. A proof of the dispersion relation

$$
\omega^{2}=k^{2} \rho_{0}\left(1-\frac{k^{2} \beta^{2}}{\rho_{0}}\right)=k^{2} \rho_{0}-k^{4} \beta^{2}
$$


for the system (5) is given in [20]. There it is also shown that this dispersion relation coincides exactly with the dispersion relation for the RNLS Equation (1), for a linearization of the latter equation about the ground state (condensate) solution

$$
\psi_{0}(x, t) \stackrel{\text { def }}{=} a_{0} \mathrm{e}^{i \gamma a_{0}^{2} t}, a_{0} \in \mathbb{R}
$$

of it, for the choice $\rho_{0}=-2 \lambda a_{0}^{2}>0$. The dispersion relation for Equation (1) is stated (without proof) in [6], for $\gamma=-1 / 2$.

\section{Conclusions}

The focal points of interest of this paper have been the nonlinear system (5), which describes the propagation of magnetoacoustic waves in a cold plasma in the presence of a transverse magnetic field, the particular traveling wave solution $(\rho, u)$ of this system given by Gurevich-Krylov in (10), and the construction of new solutions of other nonlinear systems-elliptic function solutions that we have in fact expressed in terms of the solution $(\rho, u)$. These other nonlinear systems considered were that of a Madelung fluid, a reaction diffusion system (which played a distinct, unifying role throughout the paper), systems of Broer-Kaup, Boussinesq, Hamilton-Jacobi-Bellman, and the Jackiw-Teitelboim system of gravitational field equations. An elliptic function solution of the resonance nonlinear Schrödinger Equation (1) is also expressed in terms of the solution $(\rho, u)$ in Equation (11) and Equation (15).

Regarding the gravitational field equations (see (64)), in particular, some black hole solutions $\left(g_{\text {plasma }}, \Phi_{\text {plasma }}^{(j)}, \Lambda=-2 B\right.$ ) were presented where $g_{\text {plasma }}$ is a pseudo-Riemannian metric (given in (67)) of constant Ricci scalar curvature $4 B\left(B \stackrel{\text { def }}{=}-\gamma / \beta^{2}\right.$ for $\gamma$ and $\beta^{2}$ in (1) and (5)), the $\Phi_{\text {plasma }}^{(j)}, j=1,2,3$ are dilaton fields (given by (86), (88), (94), (100), (102)), and $\Lambda$ is a (negative) cosmological constant. To obtain these solutions we used a fundamental result in [10] where an explicit change of variables was set up (which is given in definition (82) here) that transforms $g_{\text {plasma }}$ precisely to the Jackiw-Teitelboim black hole metric $g_{b h}$ in (66), for the values of $m$ and $M$ there given in (83).

Brief remarks in Section 5 further narrate a nexus between the magnetoacoustic system (5) and the resonance NLS Equation (1) - this by way of the dispersion relation (104).

The results presented here, generally speaking, provide for a non-trivial extension of a few selected results obtained in [11] [12], for example. On the other hand, as pointed out in Section 3, we have not considered an extension in the direction of two-soliton solutions of Broer-Kaup and Boussinesq systems, for example, as discussed in [11]; also see [5] [6] [16].

\section{Acknowledgements}

This paper, like that of reference [10], has drawn inspiration from the work of the authors in the references [5] [7], and [21]. 


\section{Conflicts of Interest}

The authors declare no conflicts of interest regarding the publication of this paper.

\section{References}

[1] Taniuti, T. and Washimi, H. (1996) Physical Review Letters, 17, 996-998. https://doi.org/10.1103/PhysRevLett.17.996

[2] Gardner, C.S. and Morikawa, G.K. (1960) Similarity in the Asymptotic Behavior of Collision-Free Hydromagnetic Waves and Water Waves. Courant Institute of Mathematical Sciences Report No. NYO 9082 (Unpublished).

[3] Jeffrey, A. (1973) The Quarterly Journal of the Royal Astronomical Society, 14, 183-189.

[4] Berezin, Y.A. and Karpman, V.I. (1964) Soviet Physics Journal of Experimental \& Theoretical Physics, 19, 1265-1271.

[5] Lee, J.-H., Pashaev, O.K., Rogers, C. and Schief, W.K. (2007) Journal of Plasma Physics, 73, 257-272. https://doi.org/10.1017/S0022377806004648

[6] Lee, J.-H. and Pashaev, O.K. (2007) Theoretical and Mathematical Physics, 152, 991-1003. https://doi.org/10.1007/s11232-007-0083-3

[7] Clarkson, P.A. and Rogers, C. (2018) Journal of Nonlinear Mathematical Physics, 25, 247-261. https://doi.org/10.1080/14029251.2018.1452672

[8] Gurevich, A.V. and Krylov, A.L. (1988) Doklady Akademii Nauk SSSR, 298, 608-611.

[9] Chandrasekharan, K. (1985) Elliptic Functions, Grundlehren der mathematischen Wissenschaften, Vol. 281. Springer-Verlag, Berlin. https://doi.org/10.1007/978-3-642-52244-4

[10] Williams, F. (2019) Advances in Mathematical Physics, 2019, Article ID: 4810904. https://doi.org/10.1155/2019/4810904

[11] Pashaev, O.K. (2012) Theoretical and Mathematical Physics, 172, 1147-1159. https://doi.org/10.1007/s11232-012-0103-9

[12] D’Ambroise, J. and Williams, F. (2017) Advances in Mathematical Physics, 2017, Article ID: 2154784 . https://doi.org/10.1155/2017/2154784

[13] Broer, L.J.F. (1975) Applied Scientific Research, 31, 377-395. https://doi.org/10.1007/BF00418048

[14] Kaup, D.J. (1975) Progress of Theoretical Physics, 54, 396-408. https://doi.org/10.1143/PTP.54.396

[15] Bellman, R. (1957) Dynamic Programming. Princeton Univ. Press, Princeton.

[16] Lee, J.-H. and Pashaev, O.K. (2002) Modern Physics Letters A, 17, 1601-1619. https://doi.org/10.1142/S0217732302007995

[17] Jackiw, R. (1984) A Two-Dimensional Model of Gravity. In: Christensen, S., Ed., Quantum Theory of Gravity, Adam-Hilger Ltd., Hilger, 403-420.

[18] Teitelboim, C. (1984) The Hamiltonian Structure of Two-Dimensional Spacetime and Its Relation with the Conformal Anomaly. In: Christensen, S., Ed., Quantum Theory of Gravity, Adam-Hilger Ltd., Hilger, 327-344.

[19] Williams, F. (2014) Some Selected Thoughts Old and New on Soliton-Black Hole Connections in 2d Dilaton Gravity. In: Cuevas-Maraver, J., Kevrekidis, P. and Williams, F., Eds., The Sine-Gordon Model and Its Applications. From Pendula and Josephson Junctions to Gravity and High-Energy Physics, Springer Internat. Pub., 
Berlin, 177-205. https://doi.org/10.1007/978-3-319-06722-3_8

[20] Williams, F. (2020) Remarks on the Dispersion Relation for Long Magnetoacoustic Waves in a Cold Plasma: A 2d Black Hole Connection. In: Price, L., Ed., The Wave Equation: An Overview, Nova Science Publishers, Hauppauge, 171-182.

[21] Martina, L., Pashaev, O.K. and Soliani, G. (1998) Physical Review D, 58, Article ID: 084025. https://doi.org/10.1103/PhysRevD.58.084025 